\title{
Estrategias eficaces para mejorar la adherencia a la prescripción de fármacos
}

\author{
S. Álvarez Montero, F.J. Gómez de la Fuente*, \\ P. Gallego Casado**, C. Picón García de Leániz* \\ *MIR de tercer año de Medicina de Familia y Comunitaria. Especialista en Medicina \\ de Familia y Comunitaria. Tutor de MIR de MFy C. **Diplomada \\ Universitaria en Enfermería. Centro de Salud de Torrelodones. Madrid
}

\section{RESUMEN}

Se han encontrado evidencias concluyentes en los siguientes procesos: hipertensión arterial, disli pemia, asma, infecciones agudas, epilepsia y tras tornos psicóticos.

En procesos agudos la entrega de información escrita sobre la importancia de hacer el tratamien to y con instrucciones sobre la pauta a seguir me joran la adherencia y la salud de los pacientes. In formar sobre posibles efectos secundarios no disminuyó la adherencia.

En procesos crónicos la simplificación de las pautas dosificadoras mejora la adherencia.

Otras estrategias más complejas (con mayor di ficultad para su generalización en Atención Prima ria) también han incrementado la adherencia.

Palabras clave: Adherencia. Tratamiento. Inter vención. Enfermedad.
Effective strategies to improve adherence to drug prescription

\section{ABSTRACT}

Strong evidences have been found in: high blood pressure, hyperlipidemia, asthma, acute infections, epilepsy and psychotic disorders.

For acute diseases, special counselling and written instructions on need to take all pills produce better adherence and health patient outcomes. Warnings about potential adverse effects of drugs did not worse adherence.

For chronic diseases, the simplest dose schedule produce better adherence outcomes.

More complex strategies (difficult for being ge neralized in Primary Health Care) have also pro duced better adherence outcomes.

Key words: Adherence. Treatment. Intervention. Disease.

\section{PREGUNTA ESTRUCTURADA}

¿Qué estrategias para mejorar la adherencia al tratamiento farmacológico han demostrado eficacia frente a no intervención o a intervenciones habituales?

\section{LA BÚSQUEDA}

En la librería Cochrane:

(http://www.obgyn.net/cochrane.asp): “Adherence AND treatment AND chronic disease". Se han revisado 48 títulos.
Búsqueda adicional en Medline: ((()(("patient compliance" [MeSH Terms] OR patient compliance [Text Word]) AND intervention [All Fields]) AND hasabstract [text]) AND Meta-Analysis [ptyp]) AND “human"[MeSH Terms]) AND (“1998/07/31” [PDat] : “2001/04/04”[PDat]))

\section{EL ESTUDIO}

Se trata de una revisión sistemática ${ }^{1}$ en la que se realizaron búsquedas informáticas hasta julio de 1998 en MEDLIN,CINAHL, The Cochrane Li- 
brary, International Pharmaceutical Abstracts (IPA), PsychInfo, Sociofile y HSTAR, búsquedas manuales de artículos relacionados con la adherencia, de colecciones personales de los autores de esta revisión y mediante contacto con otros autores. La última modificación sustancial se hizo en mayo de 1999 y la última actualización fue en septiembre de 2000. Se seleccionaron ensayos aleatorios controlados sin sesgos de confusión con una intervención que busque mejorar la adherencia con el tratamiento farmacológico prescrito, con al menos un seguimiento del $80 \%$ de pacientes en cada uno de los grupos estudiados y, en el caso de tratamientos crónicos con un seguimiento de al menos 6 meses para estudios con resultados iniciales positivos. La información sobre las características del estudio (diseño, grupos, hallazgos) fue realizada por varios revisores de forma independiente. Los estudios eran excesivamente dispares como para poder combinar los datos en un metaanálisis.

\section{RESULTADOS}

Ver tablas I y II.

\section{COMENTARIO}

Este artículo es una revisión sistemática rigurosa, que cumple todos los criterios exigibles a un estudio de este tipo. Incluye dos aspectos que ponen el listón de calidad de las evidencias muy alto. Se han valorado meticulosamente los sesgos de confusión, descartándose los estudios defectuosos y se han valorado sólo aquellos artículos con resultados sobre la adherencia y sobre la salud (TA, colesterol, etc). Se considera que las medidas dirigidas a incrementar la adherencia no tienen sentido en sí mismas si no mejoran resultados de salud ya que podrían dar lugar a efectos indeseables, como consumir recursos, provocar una pérdida de intimidad y autonomía en los pacientes o incrementar los efectos adversos de los medicamentos.

Como puntos débiles hay que destacar que la fecha de la última actualización relevante es de mayo de 1999, aunque se ha realizado algún cambio de pequeña entidad en septiembre de 2000, lo que sugiere que no es una revisión "en vía muerta". No obstante, se hizo una búsqueda adicional en Medline de otras revisiones sistemáticas o metaanálisis posteriores. Se encontró el trabajo de Roter et $\mathrm{al}^{2}$ que, sin embargo, estaba fechado con anterioridad a la última actualización de la revisión que nos ocupa. Puede que haya ensayos aleatorios de calidad posteriores que no están incluidos.

Otro aspecto que debilita las conclusiones es que la mayoría de los estudios eran demasiado peque- ños y la posibilidad de falsos negativos (error beta) podría ser alta. Además en ninguno se valoraron aspectos como la mortalidad o la morbilidad, y el seguimiento fue relativamente corto (el mayor de 18 meses).

\section{IMPLICACIONES PARA LA PRÁCTICA DIARIA}

La adherencia (o cumplimiento) puede definirse como el grado en el cual los pacientes siguen las intrucciones que se les da para realizar un tratamiento. Se han descrito muchos motivos para que se produzca una no adherencia, como la presencia de efectos adversos, instrucciones mal dadas, mala relación entre el profesional sanitario y el paciente, falta de acuerdo en cuanto a la necesidad del tratamiento o dificultades para pagarlo. El porcentaje de adherencia para la medicación suele rondar el 50\% (entre el 0 y más del 100\%) de la dosis prescrita ${ }^{3}$.

Sorprende ver que no hay un gran número de evidencias en este campo, por lo que es necesario tomar muy en consideración las pocas existentes. Las intervenciones las podemos clasificar en sencillas o complejas, así como en aquellas aplicadas en procesos agudos o a procesos crónicos. En general las estrategias complejas se caracterizan por un despliegue importante de recursos difícilmente generalizable a las consultas de Atención Primaria. Por este motivo hemos resaltado las intervenciones más sencillas que han tenido eficacia sobre patología más frecuentemente abordada en los Centros de Salud.

En procesos agudos sabemos que el consejo y la entrega de instrucciones escritas con la pauta a seguir, así como la importancia de seguirla mejoran la adherencia y los resultados sobre la salud de los pacientes. Además, sabemos que una mención a posibles efectos secundarios no va a "asustar" a los pacientes, reduciendo el cumplimiento ni la eficacia del tratamiento (suponiendo, claro, que no haya intencionalidad consciente o inconsciente para ello por parte del profesional). Parece difícil imaginar un folleto prediseñado para cada patología aguda que se atiende en Atención Primaria. Sin embargo, es posible escribir en la copia de la receta con signos grandes y legibles la dosis, el objetivo del fármaco (infección, dolor, proteccion gástrica, etc.) y, en casos especiales, los efectos secundarios (dolor abdominal en los AINEs, etc.) aconsejando verbalmente qué hacer si éstos surgen.

Parece razonable aplicar estas estrategias cuando se inicia un tratamiento nuevo en un problema crónico, o recurrir a los envases para escribir en ellos esta información en pacientes polimedicados.

Por otro lado, en procesos crónicos sabemos que la simplificación de las pautas dosificadoras mejora la adherencia, aunque no siempre las mediciones 


\begin{tabular}{|c|c|c|c|c|c|c|c|c|c|c|c|c|}
\hline $\begin{array}{l}\text { S } \\
\text { 面 } \\
\text { 昜号 } \\
\text { 岁 }\end{array}$ & $i \bar{n}$ & 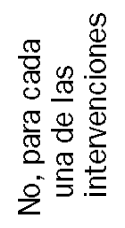 & is & $\stackrel{0}{2}$ & is & $\stackrel{2}{2}$ & is & is & 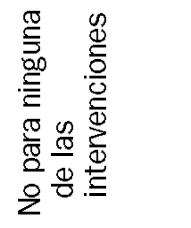 & is & 2o & is \\
\hline 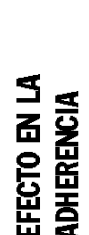 & i & 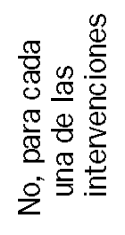 & i & $\frac{0}{2}$ & ఉ & $\frac{0}{2}$ & ๘ & ๘ & 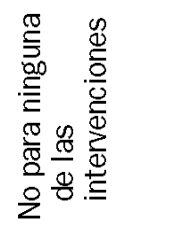 & ๘ & $\frac{0}{2}$ & is \\
\hline 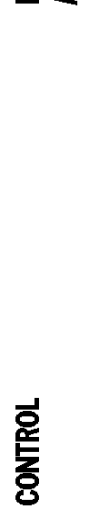 & 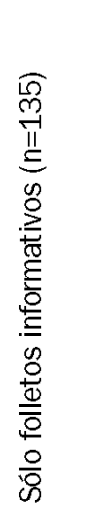 & 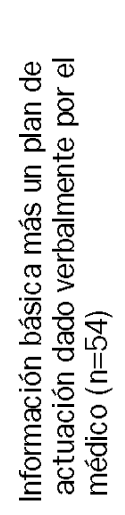 & 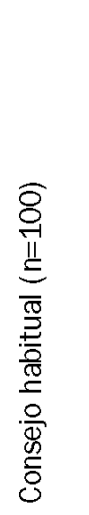 & 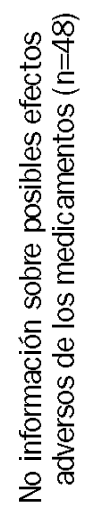 & 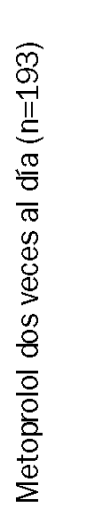 & 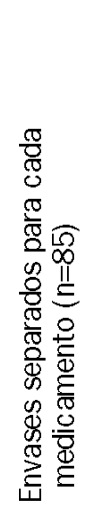 & 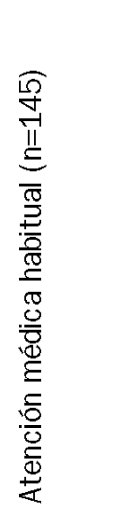 & 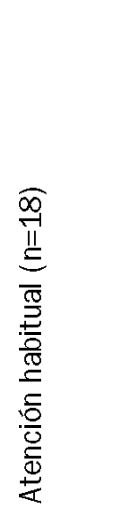 & 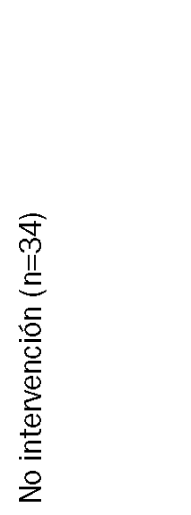 & 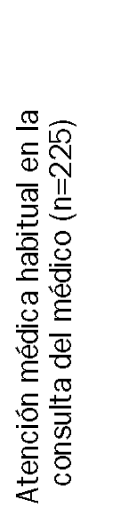 & 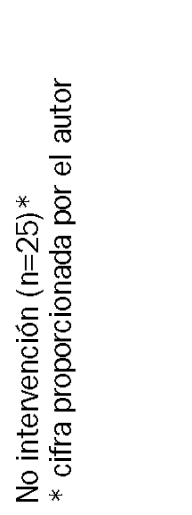 & 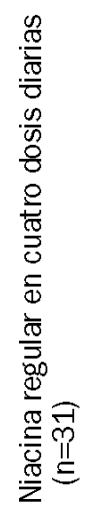 \\
\hline 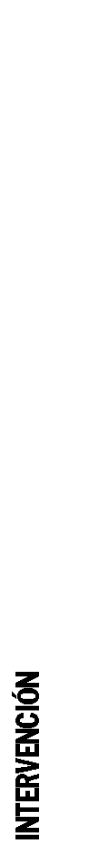 & 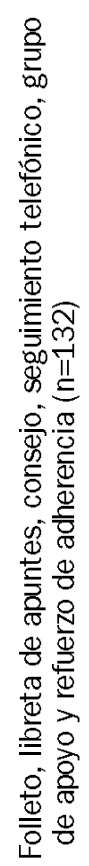 & 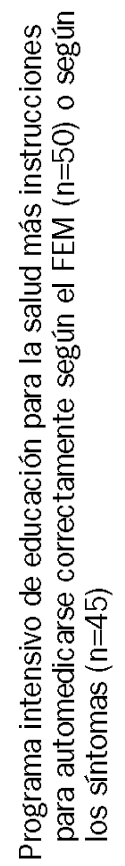 & 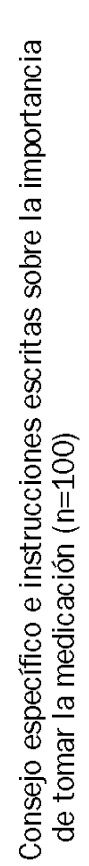 & 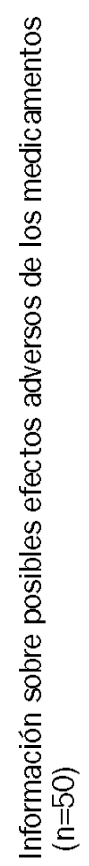 & 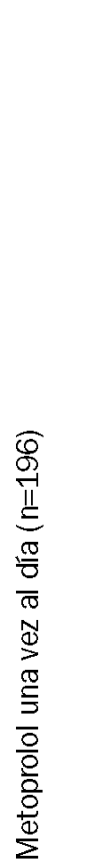 & 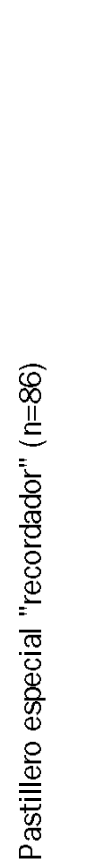 & 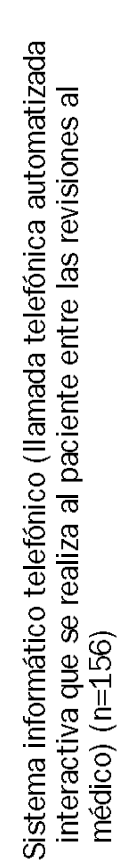 & 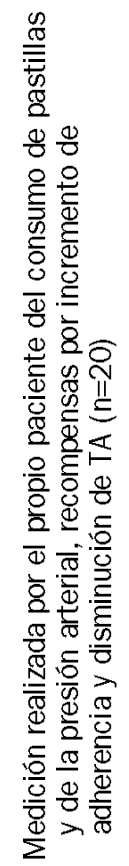 & 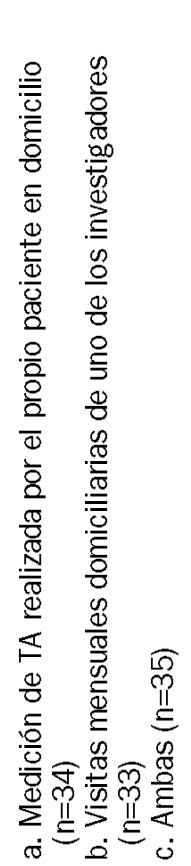 & 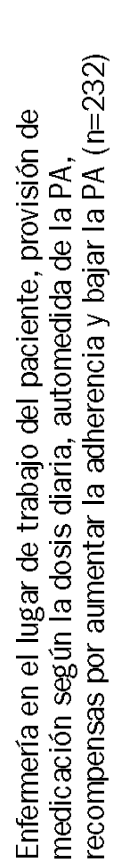 & 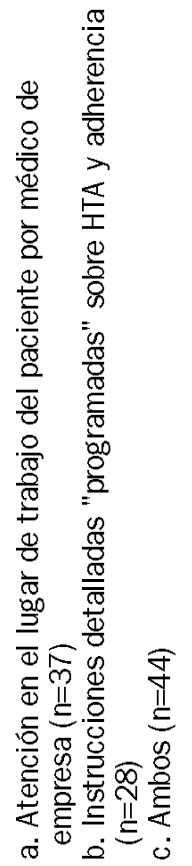 & 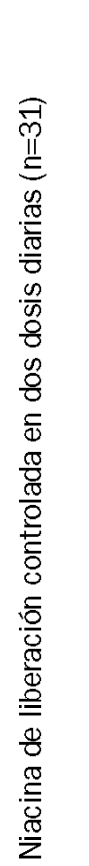 \\
\hline 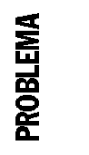 & 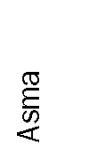 & 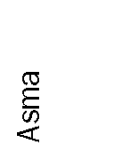 & 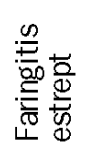 & 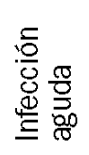 & 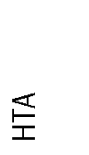 & 吉 & 泾 & 主 & 王 & 토 & 主 & 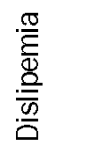 \\
\hline 음 & 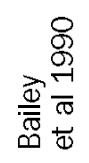 & 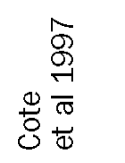 & 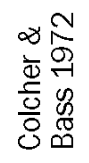 & 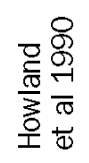 & 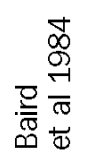 & 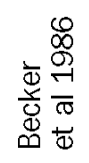 & 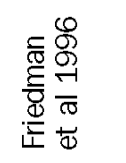 & 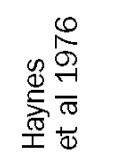 & 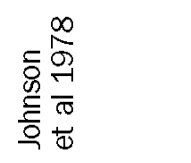 & 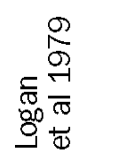 & 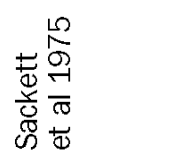 & 产 \\
\hline
\end{tabular}




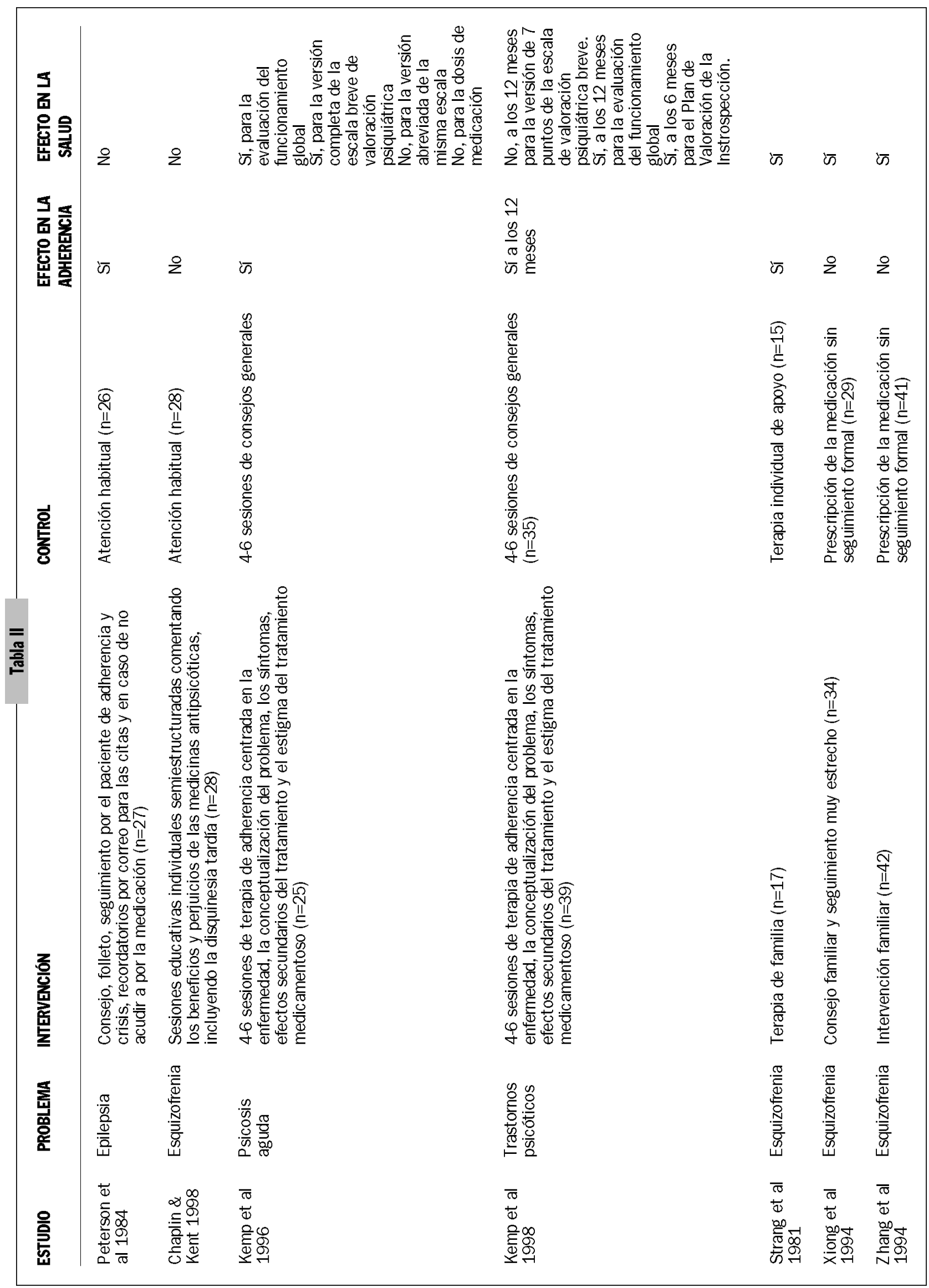


de salud. La aplicación en la práctica de estos resultados puede encarecer el coste de los medicamentos (gasto en farmacia), aunque también puede disminuir los costes totales para el sistema sanitario. Los análisis económicos son importantes para aclarar este asunto. En cualquier caso la simplificación de pautas será especialmente útil en pacientes que tengan que tomar varios medicamentos.

Fuera de esto, no hay muchas más evidencias disponibles que nos permitan llegar a conclusiones rotundas. Intervenciones muy concretas como el sistema informático telefónico no parecen aplicables a corto plazo. Hay que destacar que los pastilleros especiales no han demostrado mayor eficacia que tener cada medicamento en su envase; que los esfuerzos por dar autonomía a los pacientes (automedicación, automedida de parámetros como el flujo espiratorio o la tensión arterial); que las intervenciones de seguimiento tan estrecho como acudir a domicilio del paciente una vez al mes; o que algunas estrategias de educación para la salud bien estructuradas no mejoran necesariamente la adherencia.

El estudio de Logan et al (1979) demuestra que el seguimiento de hipertensos por personal de enfermería en el lugar de trabajo, automedición de la tensión, y recompensas por mayor cumplimiento y menores cifras de tensión, son eficaces tanto para mejorar la adherencia como la salud. Aquellos pacientes que tienen más dificultad para acceder a las consultas de Atención Primaria que al personal sanitario de su empresa pueden beneficiarse de este tipo de servicios. Las recompensas desde Atención Primaria pueden ser la disminución de visitas al Centro de Salud y la entrega de recetas sin necesidad de venir a la cita durante periodos prolongados de tiempo, siempre que se cuente con información respecto a las cifras de tensión arterial que se obtienen en el lugar de trabajo, por ejemplo a través de un familiar. Esta opción, que podría descargar de trabajo las consultas de Atención Primaria, no debería suponer una pérdida de contacto (al menos indirecto), o un desentendimiento del seguimiento del paciente.

Ante la escasez de evidencias sobre cómo mejorar el cumplimiento del tratamiento farmacológico, la opinión de los expertos cobra mayor relevancia. En este sentido los autores opinan que, dado que los pacientes que están fuera del sistema sanitario tienen muy pocas (o ninguna) probabilidades de beneficiarse de los tratamientos, y aquéllos que acuden a las consultas tienen una adherencia del $50 \%$, parece razonable pensar que cualquier esfuerzo por mantener a los pacientes dentro del sistema de atención sanitaria es la intervención más importante conocida hasta el momento. Por este motivo, estrategias como construir una relación entre el profesional sanitario y los pacientes adecuada, llamar a los pacientes que no acuden a las citas, evitar largas esperas o dificultades de contacto telefónico, pueden en el momento actual ser la mejor forma de conseguir la adherencia de los pacientes.

\section{CORRESPONDENCIA:}

Santiago Álvarez Montero

Centro de Salud de Torrelodones

Avda. de la Dehesa s/n

28250 Torrelodones. Madrid

E-mail: albaluz@teleline.es

\section{Bibliografía}

1. Haynes RB, Montague P, Oliver T, McKibbon KA, Brouwers MC, Danani R. Interventions for helping patients to follow prescriptions for medications (Cochrane Review). Oxford: The Cochrane Library 2001; lssue 1 (update software).
2. Roter DL, Hall JA, Merisca R, Nordstrom B, Cretin D, Svarsatad B. Effectiveness of interventions to improve patient compliance: a meta-analysis. Med Care 1998; 36(8): 1138-61.

3. Sackett DL, Snow JC. Compliance in Health Care. Baltimore: Johns Hopkins University Press 1979. 